\title{
Correlates of emotional intelligence among Lebanese adults: the role of depression, anxiety, suicidal ideation, alcohol use disorder, alexithymia and work fatigue
}

Sahar Obeid ${ }^{1,2,3^{*}}$, Chadia Haddad ${ }^{1,4}$, Kassandra Fares ${ }^{2}$, Diana Malaeb ${ }^{5,6}$, Hala Sacre ${ }^{3}$, Marwan Akel ${ }^{3,5}$, Pascale Salameh $\mathrm{h}^{3,7,8+}$ and Souheil Hallit ${ }^{3,9^{*}+}$ (D)

\begin{abstract}
Background: Relationship between emotional intelligence and emotional variables such as stress, depression, anxiety and mental health has been well documented in child and adult samples. New insights into the association between emotional intelligence and different components of mental health in one study (cognitive, emotional and behavioral dimensions) can help patients, therapists, relatives, and friends to understand, explain, and cope with symptoms. There have been no studies assessing the association between the emotional intelligence (EI) with various factors in Lebanon. This study principal aim was to evaluate how El is related to mental health issues: social anxiety, depression, alcohol use disorders (AUD), work fatigue, stress and alexithymia in Lebanon.
\end{abstract}

Methods: 789 participants were enrolled in a cross-sectional study between November 2017 and March 2018. A cluster analysis was used to evaluate participants' profiles with the help of emotional intelligence subscales, to separate the Lebanese population into equal limited units with different characteristics using the K-mean technique.

Results: Three clusters were computed dividing participants into low El (cluster 1; 24.5\%), moderate El (cluster 2; 43.7\%) and high El (cluster 3; 31.7\%) respectively. Fitting into the cluster 1 (low El) was significantly associated with higher AUD, alexithymia, anxiety, depression, perceived stress, social phobia, emotional, mental and physical work fatigue, suicidal ideation compared to cluster 3 (high El). Fitting into the cluster 2 (moderate El) was significantly correlated with higher AUD, depression, alexithymia, anxiety, perceived stress, social phobia, mental work fatigue and suicidal ideation compared to cluster 3 (high El).

Conclusion: This study results suggest that emotional intelligence is related to different variables, warranting interventions to limit/decrease alcohol abuse and mental/psychological illnesses as much possible.

Keywords: Emotional intelligence, Depression, Anxiety, Suicidal ideation, Alcohol use disorder, Alexithymia, Lebanon

*Correspondence: saharobeid23@hotmail.com; souheilhallit@hotmail.com ${ }^{\dagger}$ Pascale Salameh and Souheil Hallit are last co-authors

${ }^{1}$ Research and Psychology Departments, Psychiatric Hospital of the Cross, P.O. Box 60096, Jal Eddib, Lebanon

${ }^{9}$ Faculty of Medicine and Medical Sciences, Holy Spirit University of Kaslik (USEK), Jounieh, Lebanon

Full list of author information is available at the end of the article

\section{Background}

Emotional Intelligence (EI) has been described many times in psychological theories. Olivier Serrat (2017) describes EI as "the ability, capacity, skill, or self-perceived ability to identify, assess, and manage the emotions of one's self, of others, and of groups". Lyusin [1] also mentioned that feelings and the derivative 
knowledge of emotions help mankind react better with others. This idea permits individuals to define emotions, understand the importance that imposes one sentiment to another, and convert that information as a source of decision-making [1]. Petrides and Furnham believe that EI is multifactional, meaning that EI is more of a social or character variable rather than a cognitive one that is all about information handling and collecting $[2,3]$. Emotional intelligence is commonly defined by four attributes [4]: (1) Self-management-ability to control impulsive feelings and behaviors, to manage emotions in healthy ways; (2) Self-awareness-ability to recognize our own emotions and how they affect thoughts and behavior; (3) Social awareness-capacity to have empathy; (4) Relationship management/social skills- skills to develop and maintain good relationships, communicate clearly, inspire and influence others, and manage conflict. EI is thus an important parameter that affects all aspects of a person's life and conceptualizes the perception, processing, regulation, and management of emotions in self and others [5].

The concept of EI has grown quickly in recent years and has a strong relationship with mental health [6]. Thereby, the intelligent use of emotions is considered essential for one's physical health and psychological adaptation [7]. Woolery and Salovey (2004) have projected EI as a potential risk factor or protective factor in mental health [7]. Among reported negative consequences, it appears that deficiency in emotional intelligence can have a variety of detrimental psychosocial and physical outcomes for individuals that may require therapeutic intervention. Various research have studied the relation between EI and physical/mental health $[8,9]$, between emotional intelligence and emotional variables such as depression and anxiety $[10,11]$; higher emotional intelligence leads to positive quality of life while people with low emotional intelligence tend to develop psychopathology and mental health problems [12]. However, there are no studies that have taken multiple components of mental health in one research.

At the level of depression, Downey et al. witnessed connections between emotional management and control and depression severity [13]. This outcome supported the concept linking both the deficiency of emotional control and the failure to adjust feelings to clinical depression $[14,15]$. In addition, Generalized Social Phobia (GSP) is described as an evident fear of numerous social engagements. Individuals with GSP exhibit atypical neural responses to emotional stimuli and regularly show analysis bias in such circumstances. This standpoint may have proposed that GSP implicates difficulties in correctly remarking, understanding, using and handling sentiments [16].
As for alcohol use disorders (AUD), the lack of EI has been identified as a potential correlate of alcohol abuse in adolescents and adults [17]. Actually, studies have shown that inter- and intrapersonal emotional decoding deficits are present in individuals with AUD [18]. In 2007, Goldstein et al. [19] published an article correlating AUD with antisocial syndrome, leading to major relationship problems. It is for this reason that treatment of the regulation of emotions may be in favor of reducing relapses [20,21].

Previous research $[22,23]$ has also shown the existence of an adverse and noteworthy relationship between EI and exhaustion. In other words, subjects who have a high level of emotional intelligence are socially active, suffer less body pain, and are therefore less likely to develop burnout syndrome. As for stress, previous research [24, 25 ] indicated that subjects with high emotional intelligence have a great ability to regulate and express their own emotions, as well as an ability to decode the emotional states of others. Thus, the probability of subjects being overpowered by stress and developing mental health problems is low [26]. Finally, studies stated a negative association between EI and alexithymia [15, 27] and positively associated with self-esteem [28, 29].

Given the theoretical importance of EI in predicting psychological adjustment, it is expected to find a strong correlation between emotional intelligence and general psychological well-being [30, 31]. Relationship between emotional intelligence and emotional variables such as stress, depression, anxiety and mental health has been well documented in child and adult samples [11, 32]. In fact, people with higher emotional intelligence demonstrated less subjective stress, experienced better wellbeing, and demonstrated better management ability [33]. In addition, it has been demonstrated the value of EI in mood health, stress, psychosomatic health [34] and psychological well-being [35]; thus, the importance of EI depends on its ability to predict good and bad events of life. Therefore, new insights into the association between emotional intelligence and different components of mental health in one study (cognitive, emotional and behavioral dimensions) can help patients, therapists, relatives, and friends to understand, explain, and cope with symptoms. So far, research has pointed out that regulating emotions is the most important dimension of emotional competence affecting mental health. Therefore, the ability to understand emotional information is another crucial factor in daily functioning health and may be a therapeutic goal aimed at developing new programs that incorporate interventions to improve emotional skills [36].

Culturally, Ang et al. proposed that a person might be emotionally intelligent in one culture, but not in another [37]. The values and standards of each person decide the central importance in one self's lifetime and affect 
the approach in which emotions are used, identified and assessed [37]. Meta-analytical proof also proposes that traditional values and beliefs have an impact on feelings, insights and intellectual schemas [38]. This suggests that the cultural background influences the EI and can therefore be considered as a precursor of EI. For example, Yurie Igarashi [39] compared two cultural factors: individualism in America and collectivism in India. Results suggested that an individual tendency to individualism is linked to happiness experience in the first country. While an individual tendency to collectivism is linked to happiness experience in the second country [39]. Furthermore, collectivism seems to be positively linked with the use of emotions, the regulation of emotions and the emotional appraisal of others [40]. Lebanon presents a collectivistic culture where citizens are expected to interconnect with the society peers. Consequently, individual emotions are recognized and yet controlled for the profit of the unit.

On October 17, 2019, mass protests swept across Lebanon shortly after the government announced new tax measures. In a unique scene, tens of thousands of peaceful protesters from different religious and class sectors of society assembled in cities across the country accusing the political leadership of corruption and calling for social and economic reforms. Underlying frustration with the government and the political elite had been accumulating for years. Public anger has escalated in recent years over electricity and water shortages, as well as the government's failure to manage the country's waste and economic crises [41]. With the deterioration of the economic, social, political situation, protests, the high rate of poverty and unemployment, it would be important to emphasize the capacities of the Lebanese to manage their own emotions and those of others, and to understand how this management could influence their mental health. As far as we know, there have been no studies assessing the association between the emotional intelligence with various factors in Lebanon. This study principal aim was to establish the profiles of the participants in terms of EI and evaluate the association between EI and mental health issues: social anxiety, depression, alcohol use disorders (AUD), suicidal ideation, burnout, stress and alexithymia in Lebanon.

\section{Methods}

\section{Sampling and data collection}

This study had a cross-sectional design and was conducted between November 2017 and March 2018. Lebanon has two main levels of administrative division: the Mohafazat (district) and the caza (sub-district), which are divided into numerous villages. From a list provided by the Central Agency of Statistics in Lebanon, we chose two villages per Caza where survey documents were given to residents; the latter were arbitrarily selected in a proportionate manner [42]. All adults living in the house were entitled to participate. Those who agreed to take part in the study were invited to complete the questionnaire via a face-to-face interview. Excluded were those with dementia (as reported by a family member). Data assembly was completed by a personnel not related to the study team. The methodology used in this study is similar to the one used in prior publications from the same project [43-50]. The objectives of the papers from this project were different, so were the analyses and the conclusions of each paper. This is the first paper that has the objective of evaluating the association between EI (after dividing participants into clusters) and mental health issues: social anxiety, depression, AUD, suicidal ideation, burnout, stress and alexithymia in Lebanon. Although these studies and the present study utilized the same data set, we have previously used the emotional intelligence variable as part of factors/clusters that would be associated with the other dependent variables (alcohol use disorder, alexithymia, depression, self-esteem, etc.), which is different from the present study's analysis where the clusters according to EI were taken as independent variables.

\section{Minimal sample size calculation}

We set at $50 \%$ the predictable occurrence of emotional intelligence among the overall population considering the lack of national studies on this subject. The version 7.2 of the Epi-info software (population survey) estimated at least sample of 384 contributors to guarantee a $95 \%$ confidence level.

\section{Questionnaire}

The questionnaire was in Arabic, Lebanon's native language. Initially, questions about socio-demographic characteristics were included, followed by a list of scales:

\section{The Quick Emotional Intelligence Self-Assessment}

It contains 4 parts: self-management, self-awareness, social awareness and relationship management/social skills. Each part is constituted of 10 interrogations. Greater scores would signify higher EI [51]. Cronbach's alpha values for each subscale were as follows: 0.888 , $0.823,0.908$ and 0.902 respectively.

\section{The Alcohol Use Disorders Identification Test (AUDIT)}

The self-report screening tool has ten elements and evaluates alcohol usage and its consumption habits [52]. Patients having a score equal to or higher than 8 were considered to reflect AUD $\left(\alpha_{\text {Cronbach }}=0.885\right)$. 
Toronto Alexithymia Scale (TAS-20)

The TAS-20 [53] usually measures alexithymia through twenty items. Higher scores indicated higher alexithymia $[54,55]\left(\alpha_{\text {Cronbach }}=0.778\right)$.

\section{Rosenberg self-esteem scale (RSES)}

A 10-element scale processes optimistic and pessimistic approaches around one self to assess self-respect [56]. Having higher points meant higher self-worth $\left(\alpha_{\text {Cronbach }}=0.733\right)$.

\section{Hamilton depression rating scale (HDRS)}

The validated Arabic version of the HDRS [57] was used in this study [58]. Having higher results designated higher depression $\left(\alpha_{\text {Cronbach }}=0.890\right)$.

\section{Hamilton anxiety scale (HAM-A)}

The HAM-A, recently validated in Lebanon [59], is a frequently used tool to indicate the level of anxiety in health care and research places [60]. It contains 14 elements, which show higher anxiety with a higher reached score $\left(\alpha_{\text {Cronbach }}=0.898\right)$.

\section{Evaluation of the Three-Dimensional Work Fatigue Inventory (3D-WFI)}

It is composed of 18 questions, each pack of 6 questions measures physical, mental and emotional work fatigue respectively [61]. Higher scores indicate higher fatigue in all 3 dimensions. In this study, the Cronbach alpha for physical work fatigue was 0.823 , for mental work fatigue 0.667 and for emotional work fatigue 0.909 .

\section{Columbia-Suicide Severity Rating Scale (C-SSRS)}

An instrument to assess suicidal thoughts and comportment. The presence of such ideas is deliberated if the participant replied by "yes" to any question of the 5 asked categories on the C-SSRS [62]. A score of 0 signifies the nonexistence of suicidal ideas $\left(\alpha_{\text {Cronbach }}=0.762\right)$.

\section{The Perceived Stress Scale (PSS)}

This tool, composed of 10 items, assesses stress within the previous month. Higher scores show higher levels of stress $\left(\alpha_{\text {Cronbach }}=0.667\right)$.

\section{Liebowitz Social Anxiety Scale (LSAS)}

In 1987, Liebowitz established a small scale [63] with the advantage of detecting social anxiety disorder. It is a valid self-report instrument [64]. Items are grouped into 2 subdivisions: 13 intend to report performance-linked anxiety while the other 11 are concerned with social occupations. Cronbach's alpha values were 0.954 for the whole scale, 0.945 for the fear subscale and 0.953 for the avoidance subscale.

\section{Translation process}

The previously noted scales underwent forward and backward translation. An expert in mental health first converted the English version into the Arabic language. Another expert translated it back to English. A comparison of both English versions helped remove inconsistencies by consensus.

\section{Statistical analyses}

We handled data analysis using the 23rd SPSS software version. Cronbach's alpha of each scale/subscale was recorded for reliability. A cluster analysis was executed to reveal characteristics of the contributing members as stated by EI subscales and divide people into groups with distinctive profiles utilizing the K-mean method. Analysis permitted for 10 iterations centering results on 0 and convergence was simply assured through a 3-cluster design (i.e. 3 dissimilar profiles). The main benefit of cluster analysis is that similar participants can be grouped together. This helps identify patterns, reveal associations, and outline structure between participants. The emergence of a clear structure out of this analysis can allow easier decision-making. A multivariate analysis of covariance (MANCOVA) was carried out to compare multiple scales scores (being taken as dependent variables) and the 3 clusters, after adjustment over confounding variables (gender, marital status, socioeconomic status, age, children number and education level). A p-value $<0.05$ showed significance.

\section{Results}

A total of 789 (83.05\%) questionnaires was filled and handed back to the interviewer out of 950 originally distributed. The average age of the participants was $30.30 \pm 12.52$ years $(45.2 \%$ females $)$. Other sociodemographic information are arranged in Table 1 . In the absence of cutoff values for each of the emotional intelligence subscales, we took the median as a cutoff point. The results showed that $380(49.4 \%)$ had good emotional awareness, $337(43.8 \%)$ had good emotional management, 388 (50.6\%) had good social emotional awareness and 406 (51.5\%) had good relationship management.

\section{Profiles of participants}

A cluster analysis, established according to the 4 EI subscales, resulted in three mutual and exclusive clusters: low EI (24.5\%), moderate EI (43.7\%) and high EI (31.7\%) (Table 2).

\section{Clusters status and correlation with the psychological scales after adjusting for covariates}

Higher alcohol use disorder, depression, anxiety and suicidal ideation were significantly found in 


\begin{tabular}{|c|c|}
\hline & Frequency (\%) \\
\hline \multicolumn{2}{|l|}{ Gender } \\
\hline Male & $423(54.8 \%)$ \\
\hline Female & $349(45.2 \%)$ \\
\hline \multicolumn{2}{|l|}{ Education level } \\
\hline Illiterate & $12(1.6 \%)$ \\
\hline Primary & $39(5.3 \%)$ \\
\hline Complementary & $52(7.0 \%)$ \\
\hline Secondary & $113(15.2 \%)$ \\
\hline University & $462(62.3 \%)$ \\
\hline Higher education & $64(8.6 \%)$ \\
\hline \multicolumn{2}{|c|}{ Socioeconomic status } \\
\hline$<1000 \$$ & $376(50.7 \%)$ \\
\hline $1000-2000 \$$ & $260(35.1 \%)$ \\
\hline$>2000 \$$ & $105(14.2 \%)$ \\
\hline \multicolumn{2}{|l|}{ Marital status } \\
\hline Single & $488(63.1 \%)$ \\
\hline Married & $236(30.5 \%)$ \\
\hline Widowed & $19(2.5 \%)$ \\
\hline \multirow[t]{2}{*}{ Divorced } & $30(3.9 \%)$ \\
\hline & Mean \pm SD \\
\hline Age (in years) & $30.30 \pm 12.52$ \\
\hline
\end{tabular}

Table 2 Classification of participants in the study sample by cluster analysis according to the emotional intelligence scales

\begin{tabular}{|c|c|c|c|}
\hline & $\begin{array}{l}\text { Cluster } 1 \\
\mathrm{~N}=188(24.5 \%)\end{array}$ & $\begin{array}{l}\text { Cluster } 2 \\
\mathrm{~N}=335(43.7 \%)\end{array}$ & $\begin{array}{l}\text { Cluster } 3 \\
\mathrm{~N}=243(31.7 \%)\end{array}$ \\
\hline Emotional awareness score & 10.39 & 19.31 & 25.28 \\
\hline Emotional management score & 10.89 & 18.82 & 28.30 \\
\hline Social emotional awareness score & 11.44 & 21.24 & 30.22 \\
\hline Relationship management score & 11.00 & 20.52 & 30.96 \\
\hline
\end{tabular}

Cluster $1=$ People with low emotional intelligence; Cluster $2=$ People with moderate emotional intelligence; Cluster3 = People with high emotional intelligence

participants with low EI (cluster1) to moderate EI (cluster 2) compared to high EI (cluster3). Higher alexithymia, perceived stress and social phobia were significantly found in participants with low (cluster 1) vs moderate (cluster 2) EI, low (cluster 1) vs high (cluster 3) EI and moderate (cluster 2) vs high EI (cluster 3). Higher emotional and mental work fatigue were significantly found in participants belonging to cluster 1 (low EI) compared to cluster 2 (moderate EI) and cluster 3 (high EI). A significantly higher physical work fatigue was found in participants belonging to cluster 1 (low EI) compared to cluster 2 (moderate EI) and cluster 3 (high EI). Self-esteem did not differ significantly between clusters $(p>0.05)$ (Table 3$)$.

\section{Association between the sociodemographic variables} and the psychological scales

Higher means AUDIT, emotional work fatigue and suicidal ideation scores were significantly found in male participants compared to females. However, a significantly higher mental work fatigue, relationship management, social emotional awareness and emotional management appeared in male participants compared to females. A significantly higher AUDIT score was found in participants with a primary and complementary compared to university education level. A significantly higher mental work fatigue, relationship management and social emotional awareness were visible in those with a primary vs university education 
Table 3 Clusters status and correlation with the psychological scales after adjusting for covariates (age, education level, gender, SES, marital status, number of kids)

\begin{tabular}{|c|c|c|c|c|c|c|c|}
\hline \multirow[t]{2}{*}{ Variable } & \multirow{2}{*}{$\begin{array}{l}\text { Cluster } 1 \text { Low EI } \\
\text { Mean (SE) }\end{array}$} & \multirow{2}{*}{$\begin{array}{l}\text { Cluster } 2 \text { Moderate } \\
\text { EI } \\
\text { Mean (SE) }\end{array}$} & \multirow{2}{*}{$\begin{array}{l}\text { Cluster } 3 \text { High El } \\
\text { Mean (SE) }\end{array}$} & \multirow[t]{2}{*}{$F$} & \multirow{2}{*}{$\begin{array}{l}\text { P-value } \\
\text { (Cluster } 1 \text { vs. } \\
\text { cluster } 2 \text { ) }\end{array}$} & \multirow{2}{*}{$\begin{array}{l}\text { P-value } \\
\text { (cluster } 1 \text { vs. } \\
\text { cluster } 3 \text { ) }\end{array}$} & \multirow[t]{2}{*}{$\begin{array}{l}\text { P-value (Cluster } \\
2 \text { vs. cluster } 3 \text { ) }\end{array}$} \\
\hline & & & & & & & \\
\hline Alcohol dependence & $12.03(0.69)$ & $12.24(0.47)$ & $7.32(0.60)$ & 22.248 & 0.807 & $<0.001$ & $<0.001$ \\
\hline Alexithymia & $52.01(0.95)$ & $55.16(0.66)$ & $48.71(0.83)$ & 18.290 & 0.007 & 0.011 & $<0.001$ \\
\hline Self-esteem & $24.46(0.24)$ & $25.04(0.17)$ & $24.85(0.21)$ & 1.871 & 0.054 & 0.244 & 0.482 \\
\hline Depression & $15.56(0.85)$ & $15.56(0.59)$ & $7.01(0.74)$ & 45.113 & 0.999 & $<0.001$ & $<0.001$ \\
\hline Anxiety & $16.38(0.84)$ & $18.02(0.58)$ & $9.26(0.73)$ & 44.045 & 0.112 & $<0.001$ & $<0.001$ \\
\hline Perceived stress scale & $18.61(0.57)$ & $20.18(0.39)$ & $16.26(0.49)$ & 18.824 & 0.024 & 0.002 & $<0.001$ \\
\hline Social phobia & $40.85(1.90)$ & $45.82(1.32)$ & $26.63(1.66)$ & 40.814 & 0.033 & $<0.001$ & $<0.001$ \\
\hline $\begin{array}{l}\text { Emotional work } \\
\text { fatigue }\end{array}$ & $21.64(0.65)$ & $16.19(0.82)$ & $15.58(0.94)$ & 20.097 & $<0.001$ & $<0.001$ & 0.637 \\
\hline Physical work fatigue & $19.52(0.51)$ & $16.03(0.64)$ & $15.48(0.73)$ & 10.586 & $<0.001$ & $<0.001$ & 0.553 \\
\hline Mental work fatigue & $22.19(0.59)$ & $15.29(0.47)$ & $9.83(0.68)$ & 92.336 & $<0.001$ & $<0.001$ & $<0.001$ \\
\hline Suicidal ideation & $0.70(0.11)$ & $0.70(0.08)$ & $0.24(0.10)$ & 7.098 & 0.964 & 0.003 & $<0.001$ \\
\hline
\end{tabular}

Independent variable is the cluster group. Covariates are: age, education level, gender, SES, marital status, number of kids. Numbers in bold indicate significant p-values. The ANOVA test was used to compare the means of all variables between the three clusters

level. Also, a significantly higher alcohol dependence and depression were found in participants with high income as compared to low income. However, a significantly higher alexithymia was found in participants with low as compared to intermediate income. A significantly higher alexithymia and perceived stress were found in single participants as compared to the married one. In addition, higher depression and anxiety scores were significantly and positively correlated with increased age (Table 4).

\section{Multivariate analysis}

The MANCOVA analysis was performed taking the scales as the dependent variables and the three clusters as an independent variable, adjusting for the covariates (sociodemographic variables).

Fitting into the first cluster (low EI) was highly related to greater AUD (Beta $=4.71)$, alexithymia $($ Beta $=3.29)$, depression $($ Beta $=8.55)$, anxiety $($ Beta $=7.11)$, perceived stress $($ Beta $=2.35)$, social phobia $($ Beta $=14.22)$, emotional $($ Beta $=0.60)$, physical (Beta $=-3.55)$ and mental work fatigue $($ Beta $=12.36)$, and suicidal ideation $($ Beta $=0.46)$ compared to cluster 3 (high EI).

Fitting into the second cluster (moderate EI) was highly related to greater AUD (AUDIT score) $($ Beta $=4.92)$, alexithymia $($ Beta $=6.44)$, depression $($ Beta $=8.55)$, anxiety $($ Beta $=8.75)$, perceived stress $($ Beta $=3.92)$, social phobia $($ Beta $=19.19)$, mental work fatigue $($ Beta $=6.90)$ and suicidal ideation $($ Beta $=0.46)$ compared to cluster 3 (Table 5).

\section{Discussion}

The present study evaluated the association of emotional intelligence with several mental health problems, including depression, alexithymia, social phobia, work fatigue, alcohol consumption problems and stress in Lebanon. Overall, people both in the low and moderate EI clusters had higher odds of AUD, alexithymia, anxiety, depression, tension, social anxiety, exhaustion and suicidal tendency compared to those with high EI.

The results of this study showed that $24.5 \%$ of the participants were classified as having low EI, 43.7\% moderate EI and $31.7 \%$ high EI. These percentages may be discussed based on the culture differences. Several studies have revealed interesting cross-cultural differences in emotionality. For example, North Americans tend to maximize experiencing positive emotions and minimize experiencing negative emotions. However, this tendency is weaker in Asian societies [65]. Oyserman, Coon, and Kemmelmeier [66] proposed that subjects of collectivistic cultures are entertained to regulate their emotional expressions so as to maintain in-group harmony. On the other hand, Markus and Kitayama [67] [67] stated that individualists are stimulated to express their feelings more directly because they do not expect others to read their minds in social exchanges. However, Scott, Ciarrochi, and Deane [68] found that individualism was associated with less skill in managing both self and others emotions. Another study revealed that collectivists inclined to have better perception of emotions in self and others, which in turn, might make them better skilled in managing emotions in both self and others [69]. Therefore, findings of our study suggest 


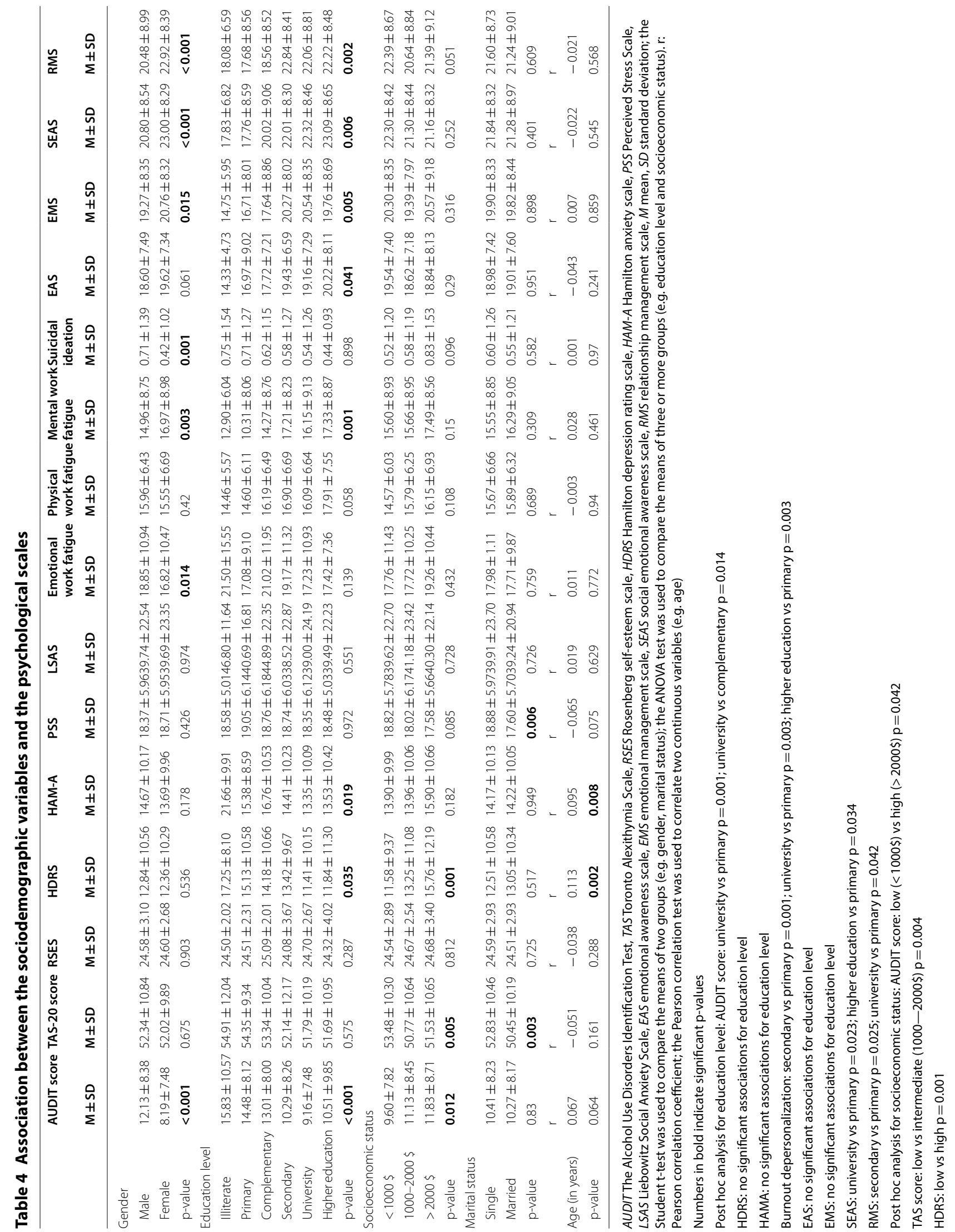


Table 5 Multivariate analysis of covariance (MANCOVA)

\begin{tabular}{|c|c|c|c|c|}
\hline & \multirow[t]{2}{*}{ Beta } & \multirow[t]{2}{*}{$p$} & \multicolumn{2}{|c|}{$\begin{array}{l}95 \% \text { confidence } \\
\text { Interval }\end{array}$} \\
\hline & & & Lower & Upper \\
\hline \multicolumn{5}{|l|}{ Alcohol use disorder (AUDIT score) } \\
\hline Gender (females versus males*) & -2.623 & $<0.001$ & -3.963 & -1.282 \\
\hline Number of kids & -1.052 & 0.012 & -1.875 & -0.229 \\
\hline University vs illiterate* level of education & -4.611 & 0.002 & -7.473 & -1.749 \\
\hline Cluster 1 (People with low emotional intelligence) vs cluster 3 (high emotional intelligence)* & 4.718 & $<0.001$ & 2.887 & 6.548 \\
\hline Cluster 2 (People with moderate emotional intelligence) vs cluster 3 (high emotional intelligence)* & 4.924 & $<0.001$ & 3.401 & 6.447 \\
\hline \multicolumn{5}{|l|}{ Alexithymia (TAS score) } \\
\hline Cluster 1 (People with low emotional intelligence) vs cluster 3 (high emotional intelligence)* & 3.299 & 0.011 & 0.764 & 5.834 \\
\hline Cluster 2 (People with moderate emotional intelligence) vs cluster 3 (high emotional intelligence)* & 6.448 & $<0.001$ & 4.339 & 8.557 \\
\hline \multicolumn{5}{|l|}{ Self-esteem } \\
\hline Cluster 1 (People with low emotional intelligence) vs cluster 3 (high emotional intelligence)* & -0.388 & 0.244 & -1.043 & 0.266 \\
\hline Cluster 2 (People with moderate emotional intelligence) vs cluster 3 (high emotional intelligence)* & 0.195 & 0.482 & -0.350 & 0.739 \\
\hline \multicolumn{5}{|l|}{ Depression } \\
\hline High vs low* SES & 4.909 & 0.039 & 0.243 & 9.575 \\
\hline Cluster 1 (People with low emotional intelligence) vs cluster 3 (high emotional intelligence)* & 8.551 & $<0.001$ & 6.291 & 10.811 \\
\hline Cluster 2 (People with moderate emotional intelligence) vs cluster 3 (high emotional intelligence)* & 8.550 & $<0.001$ & 6.670 & 10.430 \\
\hline \multicolumn{5}{|l|}{ Anxiety } \\
\hline Cluster 1 (People with low emotional intelligence) vs cluster 3 (high emotional intelligence)* & 7.113 & $<0.001$ & 4.876 & 9.350 \\
\hline Cluster 2 (People with moderate emotional intelligence) vs cluster 3 (high emotional intelligence)* & 8.754 & $<0.001$ & 6.892 & 10.615 \\
\hline \multicolumn{5}{|l|}{ Perceived Stress Scale } \\
\hline Cluster 1 (People with low emotional intelligence) vs cluster 3 (high emotional intelligence)* & 2.351 & 0.002 & 0.839 & 3.863 \\
\hline Cluster 2 (People with moderate emotional intelligence) vs cluster 3 (high emotional intelligence)* & 3.926 & $<0.001$ & 2.668 & 5.184 \\
\hline \multicolumn{5}{|l|}{ Social phobia } \\
\hline Cluster 1 (People with low emotional intelligence) vs cluster 3 (high emotional intelligence)* & 14.223 & $<0.001$ & 9.178 & 19.269 \\
\hline Cluster 2 (People with moderate emotional intelligence) vs cluster 3 (high emotional intelligence)* & 19.190 & $<0.001$ & 14.993 & 23.387 \\
\hline \multicolumn{5}{|l|}{ Emotional work fatigue } \\
\hline Cluster 1 (People with moderate emotional intelligence) vs cluster 3 (high emotional intelligence)* & 5.456 & $<0.001$ & 3.368 & 7.544 \\
\hline Cluster 2 (People with low emotional intelligence) vs cluster 3 (high emotional intelligence)* & -0.603 & 0.637 & -3.113 & 1.907 \\
\hline \multicolumn{5}{|l|}{ Physical work fatigue } \\
\hline High vs low* SES & 2.89 & 0.023 & 0.40 & 5.38 \\
\hline Cluster 1 (People with low emotional intelligence) vs cluster 3 (high emotional intelligence)* & 3.550 & $<0.001$ & 1.597 & 5.502 \\
\hline Cluster 2 (People with moderate emotional intelligence) vs cluster 3 (high emotional intelligence)* & 0.490 & 0.553 & -1.134 & 2.114 \\
\hline \multicolumn{5}{|l|}{ Mental work fatigue } \\
\hline High vs low* SES & 4.00 & 0.001 & 1.66 & 6.33 \\
\hline Cluster 1 (People with low emotional intelligence) vs cluster 3 (high emotional intelligence)* & 12.360 & $<0.001$ & 10.547 & 14.173 \\
\hline Cluster 2 (People with moderate emotional intelligence) vs cluster 3 (high emotional intelligence)* & 6.900 & $<0.001$ & 5.392 & 8.408 \\
\hline \multicolumn{5}{|l|}{ Suicidal ideation } \\
\hline Cluster 1 (People with low emotional intelligence) vs cluster 3 (high emotional intelligence)* & 0.467 & 0.003 & 0.159 & 0.775 \\
\hline Cluster 2 (People with moderate emotional intelligence) vs cluster 3 (high emotional intelligence)* & 0.460 & $<0.001$ & 0.204 & 0.716 \\
\hline
\end{tabular}

Cluster 1 = People with low emotional intelligence; Cluster2 = People with moderate emotional intelligence; Cluster3=People with high emotional intelligence In the global model, the independent variable was the cluster groups and covariates were marital status, age, SES, number of kids, education level and gender * Reference group; Numbers in bold indicate significant p-values

that emotion differentiation may increase an individual's interpersonal adaptability in collectivistic cultures; behaving appropriately in interpersonal situations often requires understanding other's feelings - a stamp of an emotionally intelligent person [70]. Thus, it may be that adaptive emotional functioning is differently related to individualistic-collectivistic orientations. 
The correlation between EI and depression is well documented in the literature [13, 71, 72]. A study had found that EI is a potential risk factor for mental and physical health, especially depression [7]. People with poor EI have a lower emotion clarity and an inability to regulate their own emotional states, in contrast to people with high EI that suffer less stress and demonstrate better management performance [73]. People with low to moderate EI had an inability to control their negative emotions and are more prone to stress and depression [74].

An overlap exists between emotional intelligence and alexithymia and this correlation had been reported in literature [75]. Studies had shown that individuals with high degrees of alexithymia experience low EI [76, 77]. In other words, these individuals face difficulties in identifying emotions in the facial expressions of others and have a limited ability to think about and use emotions to cope with stressful situations. High EI is a protecting factor against social damages, as people can correctly understand, express and use their emotions in their thinking and practice, while people with low EI are confused about their feelings, do not have any ideas about their emotions and have a difficulty expressing themselves [78].

Relationship between emotional intelligence and suicidal ideation had been reported in several studies [7981]. Numerous studies had identified various factors that may increase or decrease suicidal tendencies such as among those risk factors of suicidal propensities: depression, feeling hopelessness, problems with anger and self-esteem, difficulties in problem solving and expressing emotions [82-84]. People with high emotional intelligence have the ability to cope with stressful life events and are psychologically and physically healthier than individuals with low EI [74]. Negative life experiences of people and the incapacity to tolerate those instances, increase the vulnerability for negative thoughts as well as suicidal ideation [79].

The relation between work fatigue and emotional intelligence can be explained by the fact that people with higher EI, suffer less from the aspects of this syndrome, since they are more active, have less insomnia problems and less complains from body pains and disorders [22, 23]. Social awareness clarifies work fatigue syndrome most. Those people are aware and knowledgeable with slight social tips and connections that reflect others' requests and wishes $[22,23]$. This is a powerful skill, which qualifies people for education, professions and management features and aids them to experience good and effective relationships with others. Also, this aptitude improves contact with social care facilities that plays an important role in minimizing difficulties and conflicts [23].
The findings in this study between emotional intelligence and social phobia relation can be explained by the fact that the capacity to manage and identify social situations is tightly connected to the meaning behind EI [85-88]. Thus, it is reasonable that patients suffering from social anxiety may have deficiencies in emotional intelligence levels. It is possible that social phobia is depicted by an exaggerated terror of social situations that reflects a poor aptitude to 'read' these situations, as shown by lower EI. This is in agreement with previous results showing that being socially nervous or timid may present a lower ability to explore social situations, monitor their own emotions, as suggested by an impairment in judging their social performance [85-88].

In this study, people in the low EI category were at a higher risk of stress development since emotional intelligence is highly linked with stress management, problem-solving skills, well-being and mental health [81, 89]. The majority of studies reviewed indicate a relationship between emotional intelligence, stress, coping strategies and health [24, 81]. For example, Pau et al. concluded that people with high emotional intelligence suffered less perceived stress and experienced better health and well-being [24]. In addition, Gohm et al. concluded that emotional intelligence may protect people from stress as high emotional intelligence is related to active coping and better adaptation [90]. The use of emotional intelligence concepts may, therefore, provide insights into strategies to help foster self-engagement in practice and enhance positive attitudes, while decreasing perceived stress.

In our study, there was a close relationship between alcohol use disorder and low emotional intelligence. Evolving emotional intelligence abilities are beneficial in personal life and multiple work domains since it has been recognized to be of significant importance in alcohol abuse potential with adolescents and adults. A previous study revealed that lower EI scores were associated with reduced management strategies, that in turn anticipated a person's ability to face alcohol dependence [17]. Coping strategies have been previously shown to be linked to different variables such as motivation, self-regulation, self-awareness and social skills, the deficiency of which generally considered as major mediators of alcohol dependence [91]. Consequently, one's inability to precisely convey, realize, distinguish and control emotional expressions can predict alcohol abuse.

Concerning sociodemographic characteristics and their relationship with the scales used, our results showed that female gender was associated with lower AUD compared to males. As previous studies showed, men were more prone to alcoholism than women [92, 93]. This could be related to the cultural or religious norms and values that render women less at ease in reporting embarrassing or 
prohibited behaviors. University education level was associated with lower alcohol use disorder compared to illiteracy, as reported by other findings [94]. People with low education and those who left school are more at risk for alcohol disorders and dependence [94].

\section{Clinical implications}

Due to the significant role of EI on subjects' mental health, healthcare professionals should give a special importance to the role of emotions in the life of each person, encourage avoidance programs and arrange services to learn emotional hacks. Training some of the components of EI such as interpersonal skills, interpersonal awareness, problem solving skills, methods to cope with stress can probably be a way to improve EI overall. In a practical way, the emotional skills of individuals must be specifically trained: (1) Emotional awareness training involves identifying emotional states and recognizing how particular situations can trigger mood changes that influence performance. (2) Guidance on emotional understanding includes considering the way emotional states can guess behavior and thoughts. (3) Preparation for emotional mend denotes that subjects are capable of successfully maintaining positive emotional statuses and fixing negative ones. Therefore, specific training on EI will offer individuals means to handle nervous incidents and negative emotional reactions that commonly occur when dealing with fellow workers, family and partners. This will certainly influence their well-being and quality of life. Future research should evaluate the effectiveness of such strategies/trainings.

\section{Limitations and strengths}

Our study limitations are mostly due to the design of the cross-sectional questionnaire. Larger longitudinal schemes should examine causal sequential relationships while bearing in mind other physical, cognitive, behavioral and sociodemographic factors. Although our sample was randomly selected across Lebanese regions, a selection bias might still exist, which further hinders the generalizability of the results. In addition, the Arabic versions of different scales used have not been validated yet (the C-SSRS scale [95] and the AUDIT scale [96] have been validated among Lebanese adolescents but not adults). However, the project sheds light on various significant outcomes and is the first, as far as we know, to address factors related to emotional intelligence among a descriptive sample composed of Lebanese participants. In other words, this study was able to show an association with EI, including the mental health multicomponent in one study, and results of this study are original, in a way that they replicate the results of other Western countries.

\section{Conclusion}

In conclusion, our study found that there is a link between social anxiety, alcohol dependence, stress, selfesteem, depression, alexithymia, burnout syndrome, and emotional intelligence. This study is very useful as it has demonstrated that emotional intelligence is related to different variables, warranting interventions to limit alcohol abuse, eliminate stress, which will have positive effects on mental health. In future perspectives, we recommend that after applying the intervention strategies to a given population, it would be interesting to reevaluate the results in order to emphasize the feasibility and effectiveness of the strategies implemented.

\section{Abbreviations \\ El: Emotional intelligence; GSP: Generalized social phobia; AUD: Alcohol use disorders; AUDIT: Alcohol use disorders identification test; TAS: Toronto Alexithymia Scale; RSES: Rosenberg Self-Esteem Scale; HDRS: Hamilton Depres- sion Rating Scale; HAM-A: Hamilton Anxiety Scale; 3D-WFI: Three-dimensional work fatigue inventory; C-CSSRS: Columbia-Suicide Severity Rating Scale; PSS: Perceived Stress Scale; LSAS: Liebowitz Social Anxiety Scale; MANCOVA: Multivariate analysis of covariance.}

\section{Acknowledgements \\ The authors would like to thank all the participants who helped us during this project.}

\section{Authors' contributions}

SO and SH designed the study; MZ drafted the manuscript; $\mathrm{SH}, \mathrm{CH}$ and PS carried out the analysis and interpreted the results; $\mathrm{RH}, \mathrm{MS}$ and $\mathrm{HS}$ assisted in drafting and reviewing the manuscript; All authors reviewed the final manuscript and gave their consent. All authors read and approved the final manuscript.

\section{Funding \\ None.}

Availability of data and materials

The authors do not have the right to share any data information as per the ethics committee.

\section{Ethical approval and consent to participate}

The Psychiatric Hospital of the Cross Ethics and Research Committee, in compliance with the Hospital's Regulatory Research Protocol, approved this study protocol (HPC-012-2019). A written consent was obtained from the participants prior to starting the data collection.

\section{Consent for publication}

Not applicable.

\section{Competing interests}

The authors declare that they have no competing interests.

\section{Author details}

${ }^{1}$ Research and Psychology Departments, Psychiatric Hospital of the Cross, P.O. Box 60096, Jal Eddib, Lebanon. ${ }^{2}$ Faculty of Arts and Sciences, Holy Spirit University of Kaslik (USEK), Jounieh, Lebanon. ${ }^{3}$ INSPECT-LB: Institut National de Santé Publique, Epidémiologie Clinique et Toxicologie - Liban, Beirut, Lebanon. ${ }^{4}$ Université de Limoges, UMR 1094, Neuroépidémiologie Tropicale, Institut d'Epidémiologie et de Neurologie Tropicale, GEIST, 87000 Limoges, France. ${ }^{5}$ School of Pharmacy, Lebanese International University, Beirut, Lebanon. ${ }^{6}$ Life Sciences and Health Department, Paris-Est University, Paris, France. ${ }^{7}$ Faculty of Medicine, University of Nicosia, Nicosia, Cyprus. ${ }^{8}$ Faculty of Pharmacy, Lebanese University, Beirut, Lebanon. ${ }^{9}$ Faculty of Medicine and Medical Sciences, Holy Spirit University of Kaslik (USEK), Jounieh, Lebanon. 
Received: 24 February 2020 Accepted: 17 January 2021

Published online: 28 January 2021

\section{References}

1. Lyusin D. Emotional intelligence as a mixed construct: its relation to personality and gender. J Russ East Eur Psychol. 2006;44(6):54-68.

2. Furnham A, Petrides KV, Sisterson G, Baluch B. Repressive coping style and positive self-presentation. Br J Health Psychol. 2003;8(Pt 2):223-49.

3. Murphy KR. A critique of emotional intelligence: what are the problems and how can they be fixed? New York: Psychology Press; 2014.

4. Goleman D. Working with emotional intelligence. New York: Bantam; 1998.

5. Arora S, Ashrafian H, Davis R, Athanasiou T, Darzi A, Sevdalis N. Emotional intelligence in medicine: a systematic review through the context of the ACGME competencies. Med Educ. 2010;44(8):749-64.

6. McKinley SK, Petrusa ER, Fiedeldey-Van Dijk C, Mullen JT, Smink DS, Scott-Vernaglia SE, et al. Are there gender differences in the emotional intelligence of resident physicians? J Surg Educ. 2014;71(6):e33-40.

7. Woolery A, Salovey P. Emotional intelligence and physical health. In: Nyklicek I, Temoshok LR, Vingerhoets A, editors. Emotional expression and health: biobehavioral perspectives on health and disease prevention, vol. 6. New York: Harwood Academic Publishers; 2004. p. 154-68.

8. Petrides KV, Pérez-González JC, Furnham A. On the criterion and incremental validity of trait emotional intelligence. Cogn Emot. 2007;21(1):26-55.

9. Laborde S, Lautenbach F, Allen MS, Herbert C, Achtzehn S. The role of trait emotional intelligence in emotion regulation and performance under pressure. Personality Individ Differ. 2014;57:43-7.

10. Mayer JD. Emotion, intelligence, and emotional intelligence. Emotion. 2001;1:232-42

11. Reinherz HZ, Paradis AD, Giaconia RM, Stashwick CK, Fitzmaurice G. Childhood and adolescent predictors of major depression in the transition to adulthood. Am J Psychiatry. 2003;160(12):2141-7. https://doi. org/10.1176/appi.ajp.160.12.2141.

12. Mavroveli S, Petrides K, Rieffe C, Bakker F. Trait emotional intelligence, psychological well-being and peer-rated social competence in adolescence. Br J Dev Psychol. 2007;25(2):263-75.

13. Downey LA, Johnston PJ, Hansen K, Schembri R, Stough C, Tuckwell V, et al. The relationship between emotional intelligence and depression in a clinical sample. Eur J Psychiatry. 2008;22(2):93-8.

14. Dawda D, Hart SD. Assessing emotional intelligence: Reliability and validity of the Bar-On Emotional Quotient Inventory (EQ-i) in university students. Personality Individ Differ. 2000;28(4):797-812.

15. Saklofske DH, Austin EJ, Minski PS. Factor structure and validity of a trait emotional intelligence measure. Personality Individ Differ. 2003;34(4):707-21.

16. Jacobs M, Snow J, Geraci M, Vythilingam M, Blair R, Charney DS, et al. Association between level of emotional intelligence and severity of anxiety in generalized social phobia. J Anxiety Disord. 2008;22(8):1487-95.

17. Riley H, Schutte NS. Low emotional intelligence as a predictor of substance-use problems. J Drug Educ. 2003;33(4):391-8.

18. Maurage $P$, de Timary $P, D^{\prime}$ Hondt $F$. Heterogeneity of emotional and interpersonal difficulties in alcohol-dependence: a cluster analytic approach. J Affect Disord. 2017:217:163-73.

19. Goldstein RB, Dawson DA, Saha TD, Ruan WJ, Compton WM, Grant BF. Antisocial behavioral syndromes and DSM-IV alcohol use disorders: results from the National Epidemiologic Survey on Alcohol and Related Conditions. Alcohol Clin Exp Res. 2007;31(5):814-28.

20. Moos RH, Moos BS. Rates and predictors of relapse after natural and treated remission from alcohol use disorders. Addiction. 2006;101(2):212-22.

21. Zywiak WH, Westerberg VS, Connors GJ, Maisto SA. Exploratory findings from the Reasons for Drinking Questionnaire. J Subst Abuse Treat. 2003;25(4):287-92.

22. Carson KD, Carson PP, Birkenmeier BJ. Measuring emotional intelligence: development and validation of an instrument. J Behav Appl Manag. 2016;2(1):810

23. Chan DW. Emotional intelligence and components of burnout among Chinese secondary school teachers in Hong Kong. Teach Teach Educ. 2006;22(8):1042-54
24. Pau A, Croucher R. Emotional intelligence and perceived stress in dental undergraduates. J Dent Educ. 2003;67(9):1023-8.

25. Tsaousis I, Nikolaou I. Exploring the relationship of emotional intelligence with physical and psychological health functioning. Stress Health J Int Soc Investig Stress. 2005;21(2):77-86.

26. Cherniss C, Adler M. Promoting emotional intelligence in organizations: make training in emotional intelligence effective. Alexandria: American Society for Training and Development; 2000.

27. Austin EJ, Saklofske DH, Egan V. Personality, well-being and health correlates of trait emotional intelligence. Personality Individ Differ. 2005;38(3):547-58.

28. Hasanvand B. The relationship of emotional intelligence with self-esteem and academic progress. Int J Psychol Behav Sci. 2012;2(6):231-6.

29. Young JF, Mroczek DK. Predicting intraindividual self-concept trajectories during adolescence. J Adolesc. 2003;26(5):586-600.

30. Goldman SL, Kraemer DT, Salovey P. Beliefs about mood moderate the relationship of stress to illness and symptom reporting. J Psychosom Res. 1996:41(2):115-28.

31. Fernández-Berrocal P, Salovey P, Vera A, Extremera N, Ramos N. Cultural influences on the relation between perceived emotional intelligence and depression. Int Rev Soc Psychol. 2005;18(1):91-107.

32. Mayer JD. Emotion, intelligence, and emotional intelligence. In: Forgas JP, editor. Handbook of affect and social cognition. Mahwah, NJ: Lawrence Erlbaum Associates, Inc.; 2001. p. 410-31

33. Slaski $\mathrm{M}$, Cartwright $\mathrm{S}$. Health, performance and emotional intelligence: an exploratory study of retail managers. Stress Health J Int Soc Investig Stress. 2002;18(2):63-8.

34. Extremera N, Fernandez-Berrocal P. Emotional intelligence as predictor of mental, social, and physical health in university students. Span J Psychol. 2006;9(1):45-51.

35. Lolaty HA, Tirgari A, Fard JH. Emotional intelligence and related factors in medical sciences students of an Iranian university. Iran J Nurs Midwif Res. 2014;19(2):203-7.

36. Hertel J, Schutz A, Lammers CH. Emotional intelligence and mental disorder. J Clin Psychol. 2009;65(9):942-54.

37. Ang S, Van Dyne L, Koh C, Ng KY, Templer KJ, Tay C, et al. Cultural intelligence: its measurement and effects on cultural judgment and decision making, cultural adaptation and task performance. Manag Organ Rev. 2007;3(3):335-71

38. Taras V, Kirkman BL, Steel P. Examining the impact of culture's consequences: a three-decade, multilevel, meta-analytic review of Hofstede's cultural value dimensions. J Appl Psychol. 2010;95(3):405.

39. Igarashi Y. Personal happiness in relation to culture. EC Psychol Psychiatry. 2016:1(4):133-44

40. Gunkel M, Schlägel C, Engle RL. Culture's influence on emotional intelligence: an empirical study of nine countries. J Int Manag. 2014;20(2):256-74.

41. Amnesty International. Lebanon protests explained. https://www.amnes ty.org/en/latest/news/2019/11/lebanon-protests-explained/. Accessed 17 Jan 2020.

42. The Central Agency of Statistics in Lebanon website. www.cas.gov.lb.

43. Obeid S, Akel M, Haddad C, Fares K, Sacre H, Salameh P, et al. Factors associated with alexithymia among the Lebanese population: results of a cross-sectional study. BMC Psychol. 2019;7(1):80.

44. Obeid S, Akel M, Haddad C, Fares K, Sacre H, Salameh P, et al. Factors associated with alcohol use disorder: the role of depression, anxiety, stress, alexithymia and work fatigue - a population study in Lebanon. BMC Public Health. 2020;20(1):245

45. Obeid S, Haddad C, Akel M, Fares K, Salameh P, Hallit S. Factors associated with the adults' attachment styles in Lebanon: the role of alexithymia, depression, anxiety, stress, burnout, and emotional intelligence. Perspect Psychiatr Care. 2019;55(4):607-17.

46. Obeid S, Haddad C, Zakhour M, Fares K, Akel M, Salameh P, et al. Correlates of self-esteem among the Lebanese population: a cross-sectional study. Psychiatr Danub. 2019;31(4):429-39.

47. Obeid S, Lahoud N, Haddad C, Sacre H, Akel M, Fares K, et al. Factors associated with depression among the Lebanese population: results of a cross-sectional study. Perspect Psychiatr Care. 2020;56:956-67.

48. Obeid S, Lahoud N, Haddad C, Sacre H, Fares K, Akel M, et al. Factors associated with anxiety among the Lebanese population: the role of 
alexithymia, self-esteem, alcohol use disorders, emotional intelligence and stress and burnout. Int J Psychiatry Clin Pract. 2020;24(2):151-62.

49. Zakhour M, Haddad C, Salameh P, Akel M, Fares K, Sacre H, et al. Impact of the interaction between alexithymia and the adult attachment styles in participants with alcohol use disorder. Alcohol. 2019;83:1-8.

50. Lahoud N, Zakhour M, Haddad C, Salameh P, Akel M, Fares K, et al. Burnout and its relationships with alexithymia, stress, self-esteem, depression, alcohol use disorders, and emotional intelligence: results from a lebanese cross-sectional study. J Nerv Ment Dis. 2019;207(8):642-50.

51. Mohapel P. The quick emotional intelligence self assessment. Accessed on 2017;14.

52. Bohn MJ, Babor TF, Kranzler HR. The Alcohol Use Disorders Identification Test (AUDIT): validation of a screening instrument for use in medical settings. J Stud Alcohol. 1995;56(4):423-32.

53. Bagby RM, Parker JD, Taylor GJ. The twenty-item Toronto Alexithymia Scale-I. Item selection and cross-validation of the factor structure. J Psychosom Res. 1994;38(1):23-32.

54. Bagby RM, Taylor GJ, Parker JD. The twenty-item Toronto Alexithymia Scale- -1 . Convergent, discriminant, and concurrent validity. J Psychosom Res. 1994;38(1):33-40.

55. Thorberg FA, Young RM, Sullivan KA, Lyvers M, Hurst C, Connor JP, et al. A confirmatory factor analysis of the Toronto Alexithymia Scale (TAS-20) in an alcohol-dependent sample. Psychiatry Res. 2010;178(3):565-7.

56. Rosenberg M. Society and the adolescent self-image. Princeton: Princeton University Press; 1965.

57. Hamilton M. A rating scale for depression. J Neurol Neurosurg Psychiatry. 1960;23:56-62

58. Obeid S, Abi Elias Hallit C, Haddad C, Hany Z, Hallit S. Validation of the Hamilton Depression Rating Scale (HDRS) and sociodemographic factors associated with Lebanese depressed patients. Encephale. 2018;44:397-402.

59. Hallit S, Haddad C, Hallit R, Akel M, Obeid S, Haddad G, et al. Validation of the Hamilton anxiety rating scale and state trait anxiety inventory a and $B$ in Arabic among the Lebanese population. Clin Epidemiol Glob Health. 2020;8:1104-9.

60. Hamilton M. The assessment of anxiety states by rating. Br J Med Psychol. 1959;32(1):50-5.

61. Frone MR, Tidwell MO. The meaning and measurement of work fatigue: development and evaluation of the Three-Dimensional Work Fatigue Inventory (3D-WFI). J Occup Health Psychol. 2015;20(3):273-88.

62. Nilsson ME, Suryawanshi S, Gassmann-Mayer C, Dubrava S, McSorley P, Jiang K. Columbia-Suicide Severity Rating Scale Scoring and data analysis guide. CSSRS Scoring Vers. 2013;2:1-13.

63. Liebowitz MR. Social phobia. Mod Probl Pharmacopsychiatry. 1987;22:141-73

64. Rytwinski NK, Fresco DM, Heimberg RG, Coles ME, Liebowitz MR, Cissell S, et al. Screening for social anxiety disorder with the self-report version of the Liebowitz Social Anxiety Scale. Depress Anxiety. 2009;26(1):34-8.

65. Kitayama S, Markus HR, Kurokawa M. Culture, emotion, and wellbeing: good feelings in Japan and the United States. Cogn Emot. 2000;14(1):93-124.

66. Oyserman D, Coon HM, Kemmelmeier M. Rethinking individualism and collectivism: evaluation of theoretical assumptions and meta-analyses. Psychol Bull. 2002;128(1):3-72.

67. Markus HR, Kitayama S. Culture and the self: Implications for cognition, emotion, and motivation. Psychol Rev. 1991;98(2):224.

68. Scott G, Ciarrochi J, Deane FP. Disadvantages of being an individualist in an individualistic culture: idiocentrism, emotional competence, stress, and mental health. Aust Psychologist. 2004;39(2):143-54.

69. Bhullar N, Schutte NS, Malouff JM. Associations of individualistic-collectivistic orientations with emotional intelligence, mental health, and satisfaction with life: a tale of two countries. Individ Differ Res. 2012;10(3):165-75.

70. Kang SM, Shaver PR, Sue S, Min KH, Jing H. Culture-specific patterns in the prediction of life satisfaction: roles of emotion, relationship quality, and self-esteem. Pers Soc Psychol Bull. 2003;29(12):1596-608.

71. Fernandez-Berrocal P, Alcaide R, Extremera N, Pizarro D. The role of emotional intelligence in anxiety and depression among adolescents. Indiv Differ Res. 2006;4(1):16-27.

72. Extremera N, Fernández-Berrocal P. Emotional intelligence as predictor of mental, social, and physical health in university students. Span J Psychol. 2006;9(1):45-51.
73. Salovey P, Stroud LR, Woolery A, Epel ES. Perceived emotional intelligence, stress reactivity, and symptom reports: further explorations using the trait meta-mood scale. Psychol Health. 2002;17(5):611-27.

74. Salovey P. Applied emotional intelligence: regulating emotions to become healthy, wealthy, and wise. In: Ciarrochi J, Forgas JP, Mayer JD, editors. Emotional intelligence and everyday life. New York: Psychology Press; 2001. p. 168-84.

75. Ghiabi B, Besharat MA. Emotional intelligence, alexithymia, and interpersonal problems. Procedia-Soc Behav Sci. 2011;30:98-102.

76. Lane RD, Lee S, Reidel R, Weldon V, Kaszniak A, Schwartz GE. Impaired verbal and nonverbal emotion recognition in alexithymia. Psychosom Med. 1996;58(3):203-10.

77. Parker JD, Taylor GJ, Bagby M. Alexithymia and the recognition of facial expressions of emotion. Psychother Psychosom. 1993;59(3-4):197-202.

78. Naghavi F, Redzuan M, Mansor M. The relationship between alexitymia and emotional intelligence. Asian Soc Sci. 2010;6(11):166.

79. Kumara HMDDP. Relationship between emotional intelligence and suicidal ideation in Sri Lanka. Felicitation volume of Prof. Kulasena Widanagamage, Faculty of Social Sciences, University of Kelaniya, Kelaniya; 2016. p 229-43.

80. Rahgozar S, Motahari AA, Zolali A. Assessing Bar-On's emotional intelligence components among normal subjects and those of having suicide trial record. Indian J Sci Technol. 2011;4(10):1391-5.

81. Ciarrochi J, Deane FP, Anderson S. Emotional intelligence moderates the relationship between stress and mental health. Personality Individ Differ. 2002;32(2):197-209.

82. Evans E, Hawton K, Rodham K. Suicidal phenomena and abuse in adolescents: a review of epidemiological studies. Child Abuse Negl. 2005;29(1):45-58.

83. Hyde T, Kirkland J, Bimler D, Pechtel P. An empirical taxonomy of socialpsychological risk indicators in youth suicide. Suicide Life-Threaten Behav. 2005:35(4):436-47.

84. Orbach I. A taxonomy of factors related to suicidal behavior. Clin Psychol Sci Pract. 1997:4(3):208-24.

85. Alden LE, Wallace ST. Social phobia and social appraisal in successful and unsuccessful social interactions. Behav Res Ther. 1995;33(5):497-505.

86. Rapee RM, Lim L. Discrepancy between self-and observer ratings of performance in social phobics. J Abnorm Psychol. 1992;101(4):728.

87. Wallace ST, Alden LE. A comparison of social standards and perceived ability in anxious and nonanxious men. Cogn Ther Res. 1991;15(3):237-54.

88. Wallace ST, Alden LE. Social phobia and positive social events: the price of success. J Abnorm Psychol. 1997;106(3):416.

89. Gerits L, Derksen JJ, Verbruggen AB, Katzko M. Emotional intelligence profiles of nurses caring for people with severe behaviour problems. Personality Individ Differ. 2005;38(1):33-43.

90. Gohm CL, Corser GC, Dalsky DJ. Emotional intelligence under stress: useful, unnecessary, or irrelevant? Personality Individ Differ. 2005;39(6):1017-28.

91. Coelho KR. Emotional intelligence: An untapped resource for alcohol and other drug related prevention among adolescents and adults. Depress Res Treat. 2012. https://doi.org/10.1155/2012/281019.

92. Hasin DS, Stinson FS, Ogburn E, Grant BF. Prevalence, correlates, disability, and comorbidity of DSM-IV alcohol abuse and dependence in the United States: results from the National Epidemiologic Survey on Alcohol and Related Conditions. Arch Gen Psychiatry. 2007:64(7):830-42.

93. Andrews G, Henderson S, Hall W. Prevalence, comorbidity, disability and service utilisation: overview of the Australian National Mental Health Survey. Brit J Psychiatry. 2001;178(2):145-53.

94. Muthoni MB, Olaly W, Sirera MA, Kem P. Impact of level of education on alcohol abuse among teachers in Nyeri County, Kenya. Elixir Psychol. 2018;120:51940-4.

95. Chahine M, Salameh P, Haddad C, Sacre H, Soufia M, Akel M, et al. Suicidal ideation among Lebanese adolescents: scale validation, prevalence and correlates. BMC Psychiatry. 2020;20(1):304.

96. Hallit J, Salameh P, Haddad C, Sacre H, Soufia M, Akel M, et al. Validation of the AUDIT scale and factors associated with alcohol use disorder in adolescents: results of a National Lebanese Study. BMC Pediatr. 2020:20(1):205

\section{Publisher's Note}

Springer Nature remains neutral with regard to jurisdictional claims in published maps and institutional affiliations. 